Bažec, H. (2018). La morfosintassi degli studenti sloveni a livello A2/B1. Italica Wratislaviensia, 9(1), $11-33$.

DOI: http://dx.doi.org/10.15804/IW.2018.09.01

\author{
Helena Bažec
}

Università del Litorale

\title{
LA MORFOSINTASSI DEGLI STUDENTI SLOVENI A LIVELLO A2/B1
}

\section{SLOVENE A2/B1 STUDENTS'MORPHOSYNTAX}

\begin{abstract}
The aim of this article is to make an error analysis based on a corpus consisting of 58 written tests in Italian on the vocational maturity exam of Slovene mother-tongue students at an A2/B1 level. The objective of the qualitative and quantitative analysis is to identify the most frequent errors in the field of morphosyntax, to find out the causes, and to compare them with the results that emerged from previous research studies. The qualitative analysis was carried out on the basis of a grid created after I had completed a contrastive study of Slovene and Italian and later adapted to the data that emerged from the corpus. The causes of errors are negative transfer from L1 and in a small percentage from other foreign languages such as English and Spanish, but also the generalisation strategy. Quantitatively, the most problematic part of speech is the article, a part of speech that Slovene does not have, followed by the erroneous form or use of verbs and prepositions. The other categories, which exceed $1 \%$ of all errors and are therefore included, are the agreement between constituents, word order, and errors related to different uses of the pronoun, adjective, noun, and adverb. This frequency of errors matches other similar research studies carried out in the past with the difference that other studies included students with different Slavic languages as the L1. This suggests that all Slavic learners have the same problems in Italian written production, therefore the first cause of morphosyntactic errors is the difference in the grammatical structure between L1 and L2.
\end{abstract}

Keywords: contrastive analysis, error analysis, morphosyntax, Italian, Slovene 


\section{INTRODUZIONE}

T o studio dell'apprendimento delle lingue seconde - termine che per definizione si usa in riferimento a qualsiasi lingua imparata accanto alla lingua madre (Gass \& Selinker, 2008, p. 7) - rientra nell'ambito della linguistica applicata, ed è osservabile a partire da diversi punti di vista. Uno di questi è monitorare la competenza linguistica considerando la lingua come un sistema suddiviso in più livelli analizzabili e governato da regole che nella produzione di qualsiasi discente - prima o poi e in misura maggiore o minore - verranno violate. L'analisi degli errori è un valido metodo che dà informazioni sul processo d'apprendimento, e si basa sul presupposto che molti di essi abbiano origine nelle differenze tra la lingua madre (L1) e la lingua seconda o straniera (L2/LS). Ma un'analisi contrastiva sistematica delle grammatiche non è in grado né di prevedere tutti gli errori né di spiegarli. Per questo motivo viene coniato il termine interlingua (Selinker, 1972), che sta per il sistema di regole nascenti della L2/LS sviluppata da ogni singolo apprendente, e che rappresenta una "grammatica" transitoria e quindi mutabile. In essa possiamo osservare la maggioranza dei livelli linguistici, come ad esempio la morfologia e la sintassi. L'interlingua è suscettibile di diverse influenze, tra cui il transfer linguistico dalla L1 o da altre $\mathrm{LS}^{1}$, e le strategie della generalizzazione e della semplificazione dovute a ipotesi sul funzionamento della L2. L'obiettivo dell'analisi degli errori è dunque "descrivere la natura dell'interlingua dello studente e confrontarla con la lingua d'arrivo" (Andorno \& Nesci, 2004, p. 38).

Nel caso dello sloveno a confronto con l'italiano sono già stati presentati degli studi, che però non sono mai stati sistematici né hanno seguito la situazione di un'intera generazione nel tempo. Citiamo per prima l'analisi di Katerin Katerinov (1980), che si concentra sugli errori commessi nella produzione di traduzioni e riassunti da studenti di italiano stranieri con diverse L1. Per quanto concerne la comparazione tra sloveno e italiano, possiamo menzionare solamente un elenco degli

1 Si veda ad esempio l'articolo di Jessner (2008) per approfondimenti sul transfer dalla terza lingua. 
errori più frequenti a livello morfosintattico e lessicale dei discenti L1 slavofoni. Prendendo sotto la lente d'ingrandimento solo quelli morfosintattici, vediamo che ai primi tre posti per frequenza si collocano gli errori di articolo, di preposizioni e dello scambio tra la forma perfetta e imperfetta dei verbi seguiti da pronomi personali, concordanza dei tempi e dei modi, morfologia del verbo, scelta dell'ausiliare ecc. Katerinov aggiunge inoltre che "se, ad esempio, uno studente tedesco ha già studiato un'altra lingua romanza, al momento dell'approccio con l'italiano sarà quasi certamente bersagliato più dall'interferenza di queste lingue che non dalla propria" (Katerinov, 1980, p. 22). Questo dato è rilevante per la presente analisi in quanto si potrebbero presupporre a priori diversi errori legati al transfer negativo da altre L2 ${ }^{2}$.

Una ricerca pubblicata nel 1990 da Nelida Milani-Kruljac analizza gli errori più frequenti nella produzione in italiano da alunni slovenofoni e croatofoni di scuole nelle zone bilingui dell'Istria slovena e croata. Nel suo elenco, fra $i$ trenta aspetti più critici vengono inseriti gli errori riguardanti vari problemi nell'uso dell'articolo, delle preposizioni e lo scambio tra perfetto e imperfetto. I primi tre posti sono occupati dagli stessi errori e anche nello stesso ordine come nello studio pubblicato da Katerinov. Da quanto emerso sarebbe quindi lecito supporre che tutti i parlanti slavofoni commettano la stessa tipologia di errori con la stessa frequenza. Gli altri errori che vengono elencati, e che nello studio di Katerinov (1980) si riuniscono nel denominatore comune 'morfologia del verbo', sono: la forma riflessiva dei verbi, l'imperativo e la forma impersonale. Nel lavoro di Milani-Kruljac la distinzione tra vari tipi di errore è molto più dettagliata; infatti, anche il numero delle categorie di errori è maggiore (trenta vs dieci di Katerinov). Tra queste troviamo anche la doppia negazione, l'alternanza tra essere e esserci, problemi con le particelle $c i$ e ne e la comparazione.

Le pubblicazioni più recenti che presentano l'analisi degli errori di L1 slovenofoni non danno un quadro generale, bensì elencano degli

2 In Slovenia è obbligatorio lo studio di almeno una LS, ma già dalle elementari è possibile scegliere anche una seconda LS, nelle zone bilingui una terza (Skela \& Sešek, 2012). 
aspetti ben precisi di transfer negativo dalla L1. Tra queste si annoverano gli articoli di Martina Ožbot (2009) e Darja Mertelj \& Mirjam Premrl (2013). Ožbot studia le differenze tra lo sloveno e l'italiano nell'ambito dei suoi corsi di traduzione con studenti universitari di italiano in Slovenia e studenti di sloveno in Italia. Si focalizza sui seguenti aspetti del livello morfosintattico: ordine dei costituenti, preposizioni, avverbi, aggettivi, pronomi, morfologia del verbo e la nominalizzazione. Mertelj \& Premrl invece presentano la sintassi della frase complessa e analizzano i connettivi e i tempi verbali nelle dipendenti implicite ed esplicite. L'obiettivo del loro contributo è osservare il transfer positivo e negativo (interferenza) da tenere presenti nella progettazione didattica.

Lo studio presentato in questo contributo intende dare un quadro generale delle peculiarità morfosintattiche nella produzione scritta di studenti a un livello a cavallo tra A2 e B1 e compararlo con i dati emersi dalle ricerche svolte precedentemente, per constatare se ci siano delle differenze o tendenze emergenti. In base a quanto riportato, le ipotesi di ricerca prevedono che 1) non ci saranno sostanziali differenze con le altre ricerche simili (Katerinov, 1980; Milani-Kruljac, 1990) pubblicate precedentemente perché 2) gli errori che vengono commessi da madrelingua slavofoni sono molto simili e anche la loro frequenza dovrebbe essere tale; 3) il maggior numero di errori è generato dalle differenze morfosintattiche tra L1 e L2 e infine 4) si troveranno fenomeni di transfer negativo legati alle terze lingue (Jessner, 2008) che vengono apprese, in particolare a lingue genealogicamente affini all'italiano (Katerinov, 1980) e all'inglese in qualità di lingua franca, che sta guadagnando terreno tra $\mathrm{i}$ giovani in Slovenia a scapito di tutte le altre $\mathrm{L}_{2} / \mathrm{LS}^{3}$. Con

3 L'inglese viene introdotto nelle scuole slovene come LS per la prima volta nel 1945/46. Da allora ha fatto passi da gigante e oggi è diventato materia obbligatoria. La sua conoscenza rappresenta una delle competenze di base, fenomeno che nel mondo viene definito con il termine english-knowing bilingualism. Le altre LS che si studiano opzionalmente sono il tedesco, il francese, lo spagnolo, l'italiano e il croato. Tra queste la più studiata è il tedesco che insieme al francese, l'italiano e il croato dal $2006 \mathrm{ad}$ oggi sta perdendo il numero di studenti, mentre lo spagnolo è l'unica lingua dove la percentuale di studenti sale. In generale la scelta di una LS come materia opzionale è in calo (Skela \& Sešek, 2012, p. 75). 
l'intento di raggiungere i sopra nominati scopi e confermare o confutare le ipotesi proposte, il presente articolo consiste di cinque capitoli. Nel capitolo 2 verrà presentato nei dettagli il campione e la metodologia usata; nel capitolo 3 verranno discussi i risultati dell'analisi qualitativa e nel capitolo 4 verrà presentata l'analisi quantitativa seguita da una comparazione con i dati emersi in passato. Nel capitolo 5 verranno riassunti i più importanti risultati e menzionati possibili spunti per eventuali ricerche in futuro.

\section{PRESENTAZIONE DEL PROGETTO}

\subsection{Corpus}

La ricerca si basa su un corpus lavorativo composto dalle prove scritte all'esame di maturità professionale nel 2016. Questa prova è composta da due parti: una lettera formale o informale (60-70 parole) e un tema libero (150-160 parole) per i quali i candidati hanno a disposizione un'ora di tempo, ed è consentito loro l'uso del dizionario. Sono state raccolte 63 prove scritte provenienti da ogni istituto professionale in cui si è svolto l'esame, di cui 4 sono state scartate in quanto il livello era talmente basso da rendere impossibile qualsiasi analisi. Nel 2016, in tre sessioni distinte, 97 candidati hanno dato l'esame di maturità professionale. Fra questi, sono stati analizzati 58 esami, ossia il $65 \%$, e quindi il campione di rilevamento garantisce la rappresentatività dell'intera popolazione.

Un altro dato che serve a contestualizzare l'analisi è il livello di padronanza dell'italiano, che è stato stimato a un $\mathrm{A} 2 / \mathrm{B}^{4}$ in base al Quadro comune europeo di riferimento per la conoscenza delle lingue. La maggior parte dei candidati studia l'italiano come L2 perché proviene da una

4 In base al progetto europeo di standardizzazione degli esami di maturità svolto in Slovenia, per la prima prova scritta bisogna ottenere il $70 \%$ dei punti ( 7 su 10$)$ per oltrepassare la soglia del livello B1, mentre per la seconda prova scritta tale percentuale si abbassa al $60 \%$, ovvero a un minimo di 12 punti su 20 in totale. Circa l' $80 \%$ di tutti i candidati all'esame di maturità oltrepassa tale soglia e riceve il certificato di conoscenza dell'italiano a livello B1 (Bitenc Peharc, 2017). 
zona bilingue situata sul confine con l'Italia, mentre una percentuale più piccola lo studia come LS 5 .

È stata scelta la produzione scritta all'esame di maturità perché può garantire le stesse condizioni per tutti i candidati e perché in questo tipo di materiale si verifica il maggior numero di errori, dato che in esso "l'atto espressivo è meno condizionato e quindi il discente pensa più a cosa dire che a come dirlo" (Katerinov, 1980, p. 25).

\subsection{Tipologia di analisi}

I dati sono stati analizzati qualitativamente e quantitativamente. L'analisi qualitativa si è focalizzata sulla competenza linguistica attraverso l'analisi degli errori morfosintattici. Questi errori sono stati individuati e classificati usando una griglia preparata precedentemente sulla base di quanto emerso dall'analisi contrastiva delle grammatiche. La griglia però è stata modificata e riadattata dopo averla sperimentata in pratica, eliminando quelle categorie che non sono state riscontrate e quelle che non hanno varcato la soglia dell' $1 \%$. Per praticità di esposizione, tutte le peculiarità ritrovate sono state raggruppate seguendo il processo di cui si serve la lingua nella sua realizzazione concreta, ossia dell'assemblaggio di unità più piccole in più grandi, il che è riassunto nella TABELLA 1 (capitolo 4). L'organizzazione dei dati parte quindi dal livello di parola, in cui gli errori sono stati classificati in base alla parte del discorso, passa per il livello di sintagma e infine si conclude al livello di frase e di periodo complesso.

Si è deciso di parlare della morfosintassi in generale, dato che i livelli di analisi si influenzano a vicenda e una distinzione netta tra fenomeni osservabili a livello solo morfologico o solo sintattico non c'è. Molti degli errori sono in realtà fenomeni di interfaccia tra i due livelli perché "la lingua è una realtà più "mescolata" di quel che la linguistica, per poter lavorare, dà l'impressione di credere. Non esistono concreti fatti di lingua che appartengano davvero a un solo livello" (Vallauri, 2013, p. 241).

5 Nel 2016 il 23,2\% dei candidati proveniva da scuole in cui l'italiano si studia come LS e il $76,8 \%$ da quelle dove si studia come L2. 
Sono state incluse nell'analisi tutte quelle formulazioni che erano inadatte a causa di un'ovvia agrammaticalità. Non è stato possibile escludere quelli che sarebbero potuti risultare solamente dei lapsus in quanto, essendo gli scritti anonimi, non si poteva ottenere un feedback da parte degli studenti. Quindi l'espressione 'errore' copre le accezioni di entrambi i termini del linguaggio specialistico introdotti da Gass e Selinker (2008) e usati con significati ben distinti: errore e sbaglio.

Partendo dalla definizione di errore come una deviazione dal sistema della lingua che non rispetta una regola del codice linguistico, si devono prendere in esame solamente le infrazioni al sistema di regole proposte dalla grammatica nei capitoli dedicati alla morfologia e alla sintassi. Si consideri l'esempio (1):

1) Penso che si potete dare dacordo che se il vostro figlio vuole giocare gli videogioche deve prima fare cualcosa per la scuola [...].

Prima di procedere all'individuazione degli errori si potrebbe anche riformulare la frase affinché si avvicini il più possibile a una variante corretta, ma senza modificarla troppo (2) per poi comparare le due varianti.

2) Penso che vi possiate mettere d'accordo che, se vostro figlio vuole giocare ai videogiochi, deve prima fare qualcosa per la scuola $[\ldots]$.

Da questa comparazione emergono errori di ortografia (dacordo, cualcosa), di semantica (si dare dacordo) e i seguenti errori morfosintattici: il vostro figlio, giocare + complemento oggetto e gli videogioche. Prendiamo l'ultimo esempio: nel sintagma nominale (SN) gli videogioche ci sono due errori, il primo è la forma dell'articolo e il secondo il genere del nome. La forma sbagliata dell'articolo è sia morfologica (flessione) come anche sintattica (accordo di costituenti all'interno del SN) e come tale si dovrebbe classificare nell'interfaccia tra morfologia e sintassi che in questo caso si sovrappongono. Il secondo errore, videogioche, è un classico transfer negativo dallo sloveno, dove il nome corrispondente è femminile. Gli errori sono quindi stati inseriti nella griglia che in seguito ha facilitato l'analisi quantitativa. Non è stata invece inclusa la mancanza del congiuntivo dato che 
in questo caso non si parla di competenza linguistica bensì di quella sociolinguistica perché l'uso dell'indicativo al posto del congiuntivo in questo tipo di frase risulta appartenente al neostandard.

\section{ANALISI DEGLI ERRORI CON SPIEGAZIONE}

Come illustrato precedentemente, per comodità di esposizione, gli errori verranno raggruppati in base alla parte del discorso a cui sono legati. Le prime classi prese in analisi saranno quelle relative al SN, seguite da quelle legate al sintagma verbale (SV).

\subsection{Nome}

I problemi riguardanti la categoria del nome si possono legare alla morfologia derivativa o a quella flessiva. Nel primo caso si tratta della coniazione di neoformazioni inesistenti, soprattutto quando alla radice viene attaccato un suffisso che con essa non si combina (*testardità, *narcista, *stupidezza ecc). Nel secondo caso si osservano due categorie flessive del nome: il genere e il numero. Il genere del nome sbagliato è quasi sempre un transfer negativo dallo sloveno: *il password (slo. geslo, $\mathrm{m}) ;$ *il mio opinione (slo. mnenje, $\mathrm{n}$ ), *le stati (slo. države, f), *il problemo (slo. problem, m), *il proposto (slo. predlog, m). L'unica eccezione è la tendenza a usare la forma maschile anche con i nomi che in sloveno sono neutri, fenomeno descritto nel capitolo 3.8.2. Errori nella formazione del plurale sono stati riscontrati solamente sporadicamente con nomi stranieri $(* i$ roboti), nomi in - io $(*$ orarii) e con plurali irregolari (p. es. *genti come plurale di persona).

\subsection{Aggettivo}

Tra gli aggettivi qualificativi (AQ) ci sono derivazioni sbagliate (*ambizionati, *antisociale, *loiale per fedele (slo. lojalen), *azienda ingegnerica, ${ }^{*}$ germanese) e alcuni problemi con la gradazione, data la comparsa di forme quali il più peggiore. Tra gli aggettivi determinativi risultano difficili nell'uso solo i possessivi e gli indefiniti. I possessivi vengono usati in modo errato nei casi in cui suo subentra a loro, mentre gli indefiniti presentano varie irregolarità, sia nella scelta dell'indefinito adatto 
(quale sport per qualche sport) che nella distinzione tra quelli con la sola forma singolare o plurale (*fare qualchi amici).

\subsection{Pronome}

I vari errori nell'uso dei pronomi personali sono i più frequenti tra tutti i tipi di pronomi, in particolare lo scambio tra $c i$ e ne, $l i$ e $g l i$, e $g l i$ e $l e$, come esemplificato negli esempi (3)-(5).

3) Senza di me non possono farci a meno.

4) Io la suggero che parla con figlio e li dira o forse prima chiede se [...].

5) Parlavo con mia figlia e gli ho risposto [...].

Spesso vengono aggiunti dei pronomi ridondanti ai verbi riflessivi (es. *mi posso fidarsi, *mi vengono sempre a trovarmi), poi si sono verificati alcuni errori con i pronomi relativi (*un periodo in quale), dimostrativi (*lui era tra quei che...) e indefiniti (*qualcuni la chiamano dipendenza).

\subsection{Articolo}

Uno dei determinanti più importanti per quest'analisi è l'articolo, perché è una parte del discorso che lo sloveno non conosce ${ }^{6}$. Gli errori osservabili si possono classificare in tre categorie: omissione, aggiunta e sbagliato accordo.

Il primo posto per frequenza è occupato dalla forma sbagliata, tra cui soprattutto lo scambio tra gli e $i\left({ }^{*} i\right.$ sport, *i obiettivi, *gli genitori), tra $i l$ e lo (*nello parco, *il zucchero), tra li e $i$ (*li ragazzi), mancanza o aggiunta dell'apostrofo con un (*un'amico, *un'caffe, *un altra storia) e l'con la forma plurale del nome ( ${ }^{*} l$ 'amicizie). A seguire sulla lista di frequenza si trova l'omissione dell'articolo (*è molto brutta cosa),

6 "Lo sloveno standard non dispone della possibilità di esprimere le categorie della determinatezza e dell'indeterminatezza con le forme dell'articolo, essendo questa parte del discorso non ancora grammaticalizzata. [...] Nello sloveno colloquiale esistono delle forme lessicali il cui status grammaticale si avvicina a quello degli articoli. Si tratta di en come forma nascente dell'articolo indeterminativo e di ta come forma usata per marcare aggettivi determinati” (Bažec, 2011, p. 4). 
articoli determinativi nella maggioranza dei casi (*tutti giovani, *con quali), preposizioni semplici e non articolate (*va con famiglia in auto). L'aggiunta dell'articolo invece è la categoria meno rappresentata che probabilmente nasce come risultato della generalizzazione e si osserva con i nomi di parentela accompagnati da un aggettivo possessivo $\left({ }^{*} i l\right.$ mio padre, *la mia figlia, *il vostro figlio, *il suo padre) e nei casi di espressioni fisse in cui l'uso ha stabilito l'assenza dell'articolo (*al patto che, *mi sento al mio agio, *nel tutto, *d'accordo sul cosa fare).

\subsection{Verbo}

Nella maggioranza dei casi gli errori si legano alla coniugazione del verbo, dove le tendenze principali sono l'ipercorrettismo, quando lo studente, per falsa analogia, usa la forma sbagliata che segue la coniugazione regolare e la applica anche ai verbi irregolari (*beverai per berrai, *conducere per condurre, * suggero per suggerisco ecc). Ci sono inoltre altri casi interessanti come ad esempio l'aggiunta della stessa desinenza due volte (*facevava per faceva), il troncamento di una desinenza (*trovanno per troveranno, *eramo per eravamo), un incrocio tra la base verbale e nominale (*loro decisono per decidono in combinazione con decisione) e un incrocio tra congiuntivo e condizionale (*potressi come risultato dell'unione di potrei e potessi). La forma *noi damo per diamo è un transfer negativo siccome il verbo dare (slo. dati) alla prima persona plurale si coniuga damo.

Rimangono sempre presenti anche gli errori nella scelta dell'ausiliare, in particolare con i verbi intransitivi (*ho arrivato, * mi sei fotografato). Partendo dalle regole, gli studenti spesso commettono errori, come nel caso del verbo fotografare. Infatti, essendo solitamente accompagnato da un pronome atono, può indurre lo studente a pensare che si tratti di una particella riflessiva, e per regola i verbi riflessivi nei tempi composti prendono l'ausiliare essere. Le forme riflessive sono inoltre problematiche quando lo stesso verbo (o almeno la traduzione che più si avvicina al suo significato) in una lingua è riflessivo, nell'altra invece non lo è. Osserviamo l'esempio (6) dove imparare viene usato come un verbo riflessivo a causa del transfer negativo dalla forma slovena naučiti se, mentre in (7) la forma riflessiva ha un significato diverso da quello 
che lo studente voleva esprimere. La formulazione scriversi viene usata erroneamente in quanto il significato voluto è 'con le amiche ci scambiamo messaggi' e non 'scrivo messaggi a me stessa'.

6) Io ti consiglio di comincare qualche corso di lingua tedescha cosi ti imparerai la lingua e incontrerai persone come te.

7) Perche sono anche io dipenda di mio telefoni, sopratutto facebook, ogni giorno mi scrivo i messeggi e parlo con le mie amiche.

Un altro tipo di errore che rispecchia le differenze tra lo sloveno e l'italiano è la scelta del tempo verbale al passato. L'aspetto verbale nelle lingue slave si esprime attraverso la forma del verbo in tutti i modi e i tempi, mentre in italiano attraverso il tempo verbale nella sfera del passato. Uno studente di italiano può quindi scegliere tra la forma perfetta (passato prossimo e remoto, trapassato prossimo) e imperfetta in base all'ottica, ovvero se l'azione viene vista nella sua globalità o parzialmente, in un solo frangente del suo svolgimento (Miklič, 2007). Però non sempre i verbi sloveni nella forma perfettiva combaciano con i tempi verbali perfetti, e quelli nella loro forma imperfettiva con l'uso del tempo verbale imperfetto. La tendenza degli studenti slovenofoni è quella di farli combaciare perfettamente, e di usare quindi i tempi verbali perfetti e imperfetti con lo stesso criterio della distribuzione dei verbi di forma perfettiva e imperfettiva. Tutto ciò risulta in formulazioni sbagliate, come ad esempio *quando siamo stati piccoli oppure *io non avevo mai questi problemi.

\subsection{Avverbio}

Spesso si nota l'omissione della particella avverbiale $c i$ nel verbo esserci $\left({ }^{*} \text { al mondo sono tanti bambini obesi }\right)^{7}$, errore molto frequente causato

7 Quando essere costituisce da solo un predicato verbale nell'accezione di 'esistere' o 'essere presente/a disposizione' si definisce con valore esistenziale (Andorno, 2003). Questo valore esistenziale è tipico del verbo esserci per cui l'unione con la particella avverbiale $c i$, avente valore locatorio, è obbligatorio. Lingue diverse si servono di strategie diverse per formare frasi "esistenziali" (dall'inglese existential sentences). Nel caso dello sloveno non c'è alcuna marca sintattica a segnalarlo e si usa 
dall'assenza di tale struttura in sloveno. Si notano inoltre alcuni casi di scambio tra la forma di aggettivo e di avverbio (*davveri amici, *guida buono).

\subsection{Preposizione}

Gli errori legati alla preposizione sono stati classificati in tre gruppi distinti: il primo comprende l'accordo ovvero l'uso della preposizione inadatta al contesto, il secondo l'omissione e il terzo l'aggiunta. Una descrizione dettagliata del problema legato al primo gruppo verrà presentata nel capitolo 3.8.2. L'omissione delle preposizioni è causata dal transfer negativo dalla L1 nei casi in cui gli studenti formano calchi sintattici (*fino dieci anni, *fino giorno d'oggi, *giocare la pallavolo) e lo stesso vale anche per l'aggiunta (*vuole aiutare a tutti). È particolarmente problematico il verbo aiutare perché la sua controparte slovena ha una reggenza diversa, per cui la questione verrà trattata nel capitolo 3.8.2.

\subsection{Livello di sintagma}

In questo capitolo verranno presentati i problemi che riguardano l'ordine dei costituenti e l'accordo. L'esposizione si concentrerà prima sul SN e in seguito sul SV.

\subsubsection{Ordine di costituenti}

L'ordine dei costituenti sbagliato generalmente non crea incomprensioni a livello comunicativo, però è difficile da debellare quando ha origini nel transfer negativo. All'interno del SN gli errori di ordine dei costituenti sono legati alla posizione dell'aggettivo qualificativo in funzione attributiva. La tendenza degli studenti è quella di posizionarli sempre prima del nome, anche quando la norma della grammatica italiana non lo prevede, creando sintagmi quali *grasse persone, *peggiore proble-

semplicemente il verbo essere (slo. biti). Per esempio la frase Nell'aula ci sono due alunni si tradurrebbe come V razredu sta dva učenca oppure Dva učenca sta v razredu (in italiano letteralmente In classe sono due alunni oppure Due alunni sono in classe). In italiano è corretta solamente la seconda variante. 
ma, *tante enumerate caratteristiche, *locale frutta, *tante diverse dipendenze, *dei liberi posti di lavoro ecc. Questa tendenza è dovuta al fatto che nello sloveno l'AQ ha solo posizione pronominale (Toporišič, 2004 , p. 559), mentre nell'italiano, in dipendenza da vari fattori, "la posizione più comune degli $\mathrm{A}$ rispetto al $\mathrm{N}$ testa è quella postnominale, che viene perciò ritenuta «canonica» dal punto di vista sintattico. Gli $\mathrm{A}[\ldots]$ possono però trovarsi anche davanti ai nomi" (Salvi \& Vanelli, 2004, p. 157).

Oltre all'AQ si è rivelata problematica la posizione dell'articolo con tutto. Spesso troviamo l'articolo definito al primo posto, quindi anche precedente tutto, per la semplice assunzione che debba avere sempre la prima posizione nel SN. Però, in base alle regole che definiscono l'ordine relativo dei determinanti, i quantificatori tutti e entrambi vengono seguiti dall'articolo definito. In particolare ci sono parecchi errori quando viene aggiunto un numerale (*nelle tutte due famiglie, *le tutte phote ecc).

Nell'ambito del SV si è rivelata problematica solamente la posizione dell'avverbio. Nello sloveno precede sempre il verbo a cui si riferisce, mentre in italiano lo segue, quindi gli studenti tendono a premetterlo al verbo (*Sempre trovo una soluzione, *il problema non si mai risolve da solo, *non sai bene parlare, *facilmente perdi ecc). Alcuni degli esempi qui riportati potrebbero anche essere considerati corretti fuori contesto, però sono stati estrapolati da frasi in cui tale ordine era inaccettabile.

\subsubsection{Accordo}

L'accordo "è un fenomeno morfosintattico per il quale in un contesto sintattico definito le parole prendono una forma specifica tra le varie che possono assumere nell'ambito del loro paradigma flessivo" (Gaeta, 2010). Gli errori si riferiscono dunque ai casi in cui non viene rispettato l'accordo di numero o genere con il nome testa (*queste tipo di dipendenza, *il tuoi soldi, *in altre nazione, *questi giorno, *tutti i giorno). Questo tipo di accordo nel SN è proprio anche allo sloveno, pertanto genera transfer positivo e di conseguenza rientra tra quelle categorie di errori che l'analisi contrastiva non è stata in grado di prevedere. 
Un caso a parte rappresentano invece quegli accordi erronei causati dall'interferenza con la L1, come ad esempio il SN *il mio opinione. In questo caso lo studente ha accordato l'articolo e il possessivo al maschile perché opinione, di genere femminile, in sloveno è neutro (slo. mnenje, $\mathrm{n}$ ) e, date le tendenze di una crescente mascolinizzazione dei nomi neutri nella L1, ${ }^{8}$ si tende ad accordare al maschile anche nella L2. Pertanto questo errore viene classificato nel gruppo del nome in base alla griglia presentata nel capitolo 4 .

Un paragrafo a parte va dedicato alla reggenza del verbo in italiano perché, non essendoci i casi, è proprio il verbo a imporre l'accordo con una determinata preposizione. Infatti, tra gli aspetti più problematici della produzione linguistica (che richiede una conoscenza approfondita della lingua) ci sono quelli legati all'idiomaticità, e questa coinvolge anche l'uso delle preposizioni (Ožbot, 2009, p. 35). Il principale problema riguardante la loro scelta ${ }^{9}$ sta nel fatto che si tende a tradurle dallo sloveno, come ad esempio far equivalere $s u=n a$ oppure $c o n=s / z$ ecc., ma la loro semantica s'interseca solo parzialmente. Il risultato è la scelta (o meglio traduzione) della preposizione in base alla reggenza del verbo in sloveno, il che porta a calchi sintattici come esemplificato in (8). La variante $a$ è quella riscontrata nel corpus e inclusa tra gli errori, la $b$ ricostruisce la reggenza slovena che sta alla base del transfer negativo e la c. rappresenta la variante italiana corretta.

8) a. Non mi arrabbio mai su qualcuno.

b. slo. jeziti se na (arrabbiare: inf. / rifl. / prep.: su)

c. Non mi arrabbio mai con qualcuno.

8 Molti nomi neutri terminano in -o atona che è stata affetta dalla riduzione vocalica moderna. L'interazione tra la coincidenza del suono nel nominativo e nell'accusativo assieme a molte desinenze in comune negli altri casi tra il neutro e il maschile hanno fatto sì che nei dialetti (e parlate) innovativi si verifichi la mascolinizzazione (Smole, 2006, p. 127).

9 Questo tipo di errore si verifica anche in relazione ad aggettivi (es. *interessati per invece di interessati a) e nomi (es. *dipendenza della tecnologia per dipendenza dalla tecnologia), ma in maniera minore. 
Nell'esempio (8) l'uso della preposizione $s u$ al posto di con è causato dalla generalizzazione prima menzionata, cioè che la preposizione slovena $n a$ equivalga in italiano a $s u$ in tutti i contesti, e quindi anche il verbo arrabbiarsi (slo. jeziti se) dovrebbe legare a sé i complementi attraverso la stessa. Tuttavia, l'accordo con la preposizione sbagliata non è dettato sempre dalla L1, in quanto nell'esempio (9) essa non ricalca la L1.

9) a. in fine di scuola

b. slo. ob koncu šole (prep.: vicino a / fine: dat. m. sg. / scuola: gen. sg. f.)

c. alla fine della scuola

Risulta altrettanto interessante la reggenza del verbo in relazione alla forma del pronome. Come sappiamo, i pronomi personali hanno una delle flessioni più articolate della lingua italiana perché oltre che per genere, persona e numero si flettono anche per caso. Possono, infatti, esprimere - oltre al nominativo - il genitivo (ne), il dativo ( $g l i$, le) e l'accusativo $(l o, l a, l i, l e)$. Le differenze tra le due lingue si fanno notare in forma di errore quando, esattamente come nel caso della scelta della preposizione, un determinato verbo in italiano lega a sé un accusativo, mentre in sloveno un dativo e viceversa. Tra questi verbi si annovera sicuramente aiutare, che in italiano richiede la forma accusativa del pronome e in sloveno quella dativa, pertanto si formano costruzioni come esemplificato in (10).

10) a. Sono sicura che lui gli sappra aiutare.

b. Prepričana sem, da mu bo on znal pomagati (sicuro: f. sg. / aus. $1^{\text {a }}$ p. sg. / cong.: che / lui: dat. / aus.: $3^{a}$ p. sg. fut. / lui: nom. / sapere: part. m. sg. / aiutare: inf.)

c. Sono sicurà che lui lo saprà aiutare.

Comparando lo sloveno con l'italiano si evince che sono problematici da questo punto di vista solo pochi verbi, perché la maggioranza di essi richiede lo stesso caso, il che fa supporre di non dover incorrere in errori legati a verbi dalla stessa reggenza. Tuttavia sono state riscontrate strutture come *tu devi darli in cui l'uso di $l i$ al posto di gli non ha ori- 
gini nella L1 degli studenti. Il verbo dare (slo. dati) di fatto ha la stessa reggenza in entrambe le lingue. Tutti gli errori legati alla forma sbagliata del pronome sono classificati nel gruppo dei pronomi (capitolo 3.3).

3.9. Livello di frase semplice e complessa

Sia lo sloveno sia l'italiano fanno concordare il soggetto e il predicato nel numero, nella persona e nel genere (participio passato). Quindi è lecito presupporre che la maggioranza degli errori non è causata dal transfer negativo (*lui frequenti la scuola, *io da bambino questi problemi non gli ho avuto ecc). Il transfer negativo dallo sloveno si verifica quando abbiamo a che fare con nomi che in una delle lingue sono difettivi e quindi si accordano solo al singolare o al plurale, come ad esempio i nomi sloveni vrata (it. porta) o usta (it. bocca) che sono dei pluralia tantum. L'accordo con questi viene calcato anche nella L2/LS e si generano frasi come p. es. *La mia bocca si rifiutano di parlare.

Nell'esempio (11), in italiano la testa del sintagma soggetto è 'giovani' e si accorda alla terza persona plurale, mentre nello sloveno il soggetto è rappresentato da veliko (molto), seguito da un nome al genitivo. Nello sloveno, veliko si comporta come i numerali da cinque in su e si accorda con la terza persona singolare, il che dà origine all'errore in (11) a.

11) a. Tanti giovani si decide

b. Veliko mladih se odloči (tanti: avv. / giovani: s. m. gen. pl. / rifl. / decidere: $3^{\circ}$ p. sg.)

c. Tanti giovani si decidono

In italiano è possibile adoperare diversi allocutivi di cortesia: per rivolgerci a un solo interlocutore si usa il 'lei' che si accorda con il verbo alla terza persona singolare, mentre per rivolgersi a più interlocutori si usa il 'voi' che si accorda con il verbo alla seconda persona plurale. Nell'italiano regionale meridionale è tutt'oggi in uso il voi come allocutivo di cortesia rivolto a un solo interlocutore. In sloveno, invece, l'unica forma di cortesia in uso è il pronome 'voi' che si accorda sempre con il verbo alla seconda persona plurale. Di conseguenza non vengono analizzati come errore i casi in cui il voi si usa con riferimento a un solo 
interlocutore, bensì quei casi in cui i pronomi Lei e Voi e le relative forme verbali si alternano, come esemplificato in (12).

12) Lei puo fare $[\ldots]$ quello che vi ho detto.

Lo sloveno non conosce il congiuntivo, e in traduzione viene reso dall'indicativo, dal condizionale, dall'imperativo o si esprime attraverso elementi lessicali in dipendenza dal tipo di frase in cui si usa (Skubic, 1974). Nelle dipendenti tende a essere sostituito dall'indicativo o dal condizionale $^{10}$. Una delle costruzioni più problematiche è il periodo ipotetico del secondo e terzo tipo in cui il congiuntivo viene rimpiazzato sistematicamente dal condizionale $\left({ }^{*}\right.$ Se io sarei lui..., *Sarebbe bello[...] se potrebbero lavorare...).

Comparando l'indicativo nelle due lingue possiamo constatare che nello sloveno ci sono solamente tre tempi verbali, rispetto agli otto dell'italiano. La sfera temporale più problematica è quella del passato, dove spesso viene seguita la concordanza dello sloveno che prevede l'uso del passato semplice (in italiano imperfetto, passato prossimo o remoto) per l'anteriorità, del presente per la contemporaneità e del futuro semplice per la posteriorità. Sono inoltre presenti diversi salti temporali a livello testuale che indicano un uso indiscriminato tra passato prossimo, passato remoto e presente storico (13).

13) Un giorno la sua madre mi chiese, perché non lo invitiamo fuori, così che almeno per un'ora non sara sul computer. Io non sappevo di cosa sta parlando, perche lui mi ha sempre detto che aveva da fare per scuola.

Un capitolo a parte va dedicato ai calchi sintattici dallo sloveno. Il primo di questi riguarda le subordinate implicite, che nello sloveno hanno una bassissima frequenza d'uso. Pertanto, si tende a scrivere solo frasi esplicite anche quando si ha identità di soggetto (*consiglierei a Corrado che andrebbe..., *preferisco che sono con quella persona ...). Questo tipo di frasi rispecchia la struttura slovena e, benché in italiano

10 Non vengono in quest'analisi considerati e commentati i tratti del neostandard, ovvero gli usi dell'indicativo al posto del congiuntivo considerati tali. 
le frasi esplicite siano corrette, non sono però consone alla tipologia testuale prodotta dagli studenti. Un altro calco dallo sloveno comprende la collocazione di vari elementi tra ausiliare e participio e tra copula e parte nominale nelle frasi dipendenti (14).

14) $[\ldots]$ sono i bambini fino ai dieci anni obesi.

Benché entrambe le lingue appartengano alla tipologia sintattica SVO, nelle realizzazioni concrete l'ordine dei costituenti si differenzia. La flessione nominale in sloveno è molto simile a quella latina, a causa della marca di caso sui nomi. Questo permette un ordine dei costituenti più libero rispetto all'italiano, perché i ruoli sintattici sono segnati dalla morfologia nominale. Se in italiano la posizione nella frase dichiarativa non marcata è determinata dal ruolo logico-semantico, nello sloveno non necessariamente è così. Infatti, una struttura tipica è l'ordine OVS senza ripresa pronominale che viene regolarmente calcata in italiano $(* i l$ computer uso per la scuola, *questo fanno tutti giovani, *tutte le ossessioni puoi finire ecc.). L'ultimo caso di struttura linguistica che nasce come calco sintattico dallo sloveno è la ripresa pronominale dopo il connettivo relativo che, come esemplificato in (15). Nello sloveno questo tipo di ripresa pronominale è obbligatorio, il che si riflette nell'italiano.

15) a. (leggendo la lettera) che l'ha scritta

b. ki jo je napisala (pron. rel.: che / pron. pers.: la / aus: $3^{\circ}$ p. sg. / scritto: f. sg.)

c. leggendo la lettera che ha scritto

Viene infine presentata una tendenza emergente che non è ancora stata registrata. Si tratta di calchi sintattici dallo spagnolo che vengono osservati in diversi candidati (*se va diventare gay, *si va como $i$ suoi genitori ecc.). Questa tendenza viene solamente menzionata e non è stata inclusa tra gli errori perché non oltrepassa $1 ' 1 \%$, come succede con i calchi dall'inglese, dal dialetto e nei casi di doppia negazione, che però sono fenomeni notati anche in precedenza. 


\section{ANALISI QUANTITATIVA E COMPARAZIONE}

Trasformando i dati empirici emersi dal corpus in percentuali, si può sostenere che con la quota del 30\% l'articolo risulta l'elemento più problematico in assoluto. Al secondo posto si trova la categoria del verbo con il 19\%, mentre gli errori relativi ai vari usi della preposizione si classificano in terza posizione (17\%). Le altre categorie si seguono nell'ordine seguente: accordo a livello di sintagma e di frase (13\%), ordine dei costituenti nel sintagma e nella frase $(8 \%)$, pronome $(6 \%)$, aggettivo $(3,5 \%)$, nome $(2 \%)$ e avverbio $(1,5 \%)$. In media ogni candidato ha accumulato 19,3 errori nell'intera prova scritta. Tutti i dettagli sulla frequenza degli errori per sottocategoria si trovano nella TABELLA 1.

Tabella 1: Griglia per l'analisi degli errori con i dati

\begin{tabular}{|c|c|c|c|c|c|}
\hline & & GENNAIO & GIUGNO & SETTEMBRE & TOTALE \\
\hline \multirow[t]{2}{*}{ Articolo } & $\begin{array}{l}\text { omissione } \\
\text { aggiunta } \\
\text { accordo }\end{array}$ & $\begin{array}{l}9 \\
2 \\
6\end{array}$ & $\begin{array}{c}92 \\
50 \\
134\end{array}$ & $\begin{array}{c}54 \\
7 \\
29\end{array}$ & $\begin{array}{c}155 \\
59 \\
169\end{array}$ \\
\hline & & 17 & 276 & 90 & 383 \\
\hline \multirow[t]{2}{*}{ Nome } & $\begin{array}{c}\text { derivazione } \\
\text { flessione: genere } \\
\text { flessione: numero }\end{array}$ & $\begin{array}{l}1 \\
5 \\
4\end{array}$ & $\begin{array}{l}0 \\
2 \\
1\end{array}$ & $\begin{array}{l}0 \\
9 \\
1\end{array}$ & $\begin{array}{c}1 \\
16 \\
6\end{array}$ \\
\hline & & 10 & 3 & 10 & 23 \\
\hline \multirow[t]{2}{*}{ Aggettivo } & $\begin{array}{c}\text { derivazione } \\
\text { gradazione } \\
\text { possessivi } \\
\text { indefiniti }\end{array}$ & $\begin{array}{l}2 \\
0 \\
0 \\
1\end{array}$ & $\begin{array}{c}15 \\
1 \\
14 \\
3\end{array}$ & $\begin{array}{l}3 \\
2 \\
2 \\
1\end{array}$ & $\begin{array}{c}20 \\
3 \\
16 \\
5\end{array}$ \\
\hline & & 3 & 33 & 8 & 44 \\
\hline \multirow[t]{2}{*}{ Pronome } & $\begin{array}{l}\text { aggiunta } \\
\text { omissione } \\
\text { personali } \\
\text { relativi } \\
\text { dimostrativi } \\
\text { indefiniti }\end{array}$ & $\begin{array}{l}0 \\
1 \\
5 \\
0 \\
0 \\
0\end{array}$ & $\begin{array}{c}6 \\
5 \\
38 \\
6 \\
2 \\
3\end{array}$ & $\begin{array}{l}1 \\
1 \\
6 \\
0 \\
0 \\
1\end{array}$ & $\begin{array}{c}7 \\
7 \\
49 \\
6 \\
2 \\
4\end{array}$ \\
\hline & & 6 & 60 & 9 & 75 \\
\hline
\end{tabular}




\begin{tabular}{c|c|c|c|c|c}
\hline & & GENNAIO & GIUGNO & SETTEMBRE & TOTALE \\
\hline \multirow{2}{*}{ Verbo } & tempi verbali & 16 & 136 & 14 & 165 \\
& coniugazione & 7 & 31 & 5 & 43 \\
& scelta ausiliare & 1 & 7 & 2 & 10 \\
& riflessivi & 6 & 12 & 3 & 21 \\
\hline \multirow{2}{*}{ Avverbio } & & 30 & 186 & 24 & $\mathbf{2 4 0}$ \\
\hline \multirow{2}{*}{ Preposizione } & esser(ci) & 3 & 7 & 7 & 17 \\
& & 3 & 7 & 7 & $\mathbf{1 7}$ \\
& agmissione & 1 & 49 & 10 & 60 \\
& accordo & 3 & 13 & 5 & 19 \\
\hline \multirow{2}{*}{ Accordo } & livello sintagma & 2 & 171 & 46 & $\mathbf{2 2 2}$ \\
& livello frase & 2 & 19 & 10 & 27 \\
& concordanza & 3 & 97 & 14 & 31 \\
\hline \multirow{2}{*}{ TOTALE } & & 7 & 131 & 34 & $\mathbf{1 7 2}$ \\
\hline \multirow{2}{*}{ Ordine costi- } & livello sintagma & 5 & 52 & 19 & 76 \\
\hline & livello frase & 11 & 25 & 8 & 44 \\
\hline & & 16 & 77 & 27 & $\mathbf{1 2 0}$ \\
\hline
\end{tabular}

Una prima comparazione con le ricerche precedenti citate nel capitolo 1 dimostra che i problemi degli slovenofoni sono condivisi dagli L1 slavofoni in generale. Andando nel dettaglio, si può notare che in tutte le ricerche al primo posto si trovano errori legati ai vari aspetti dell'uso degli articoli. Nelle prime due ricerche, al secondo posto si collocano le preposizioni e al terzo lo scambio tra la forma perfetta e imperfetta dei tempi verbali. Nella presente ricerca al secondo posto si colloca il verbo, perché include tutti i problemi a esso legati: oltre allo scambio tra la forma perfetta e imperfetta, anche la coniugazione del verbo, i verbi riflessivi e la scelta dell'ausiliare, che nelle altre ricerche comportavano categorie autonome. Al terzo troviamo le preposizioni, analogamente alle altre ricerche. Vengono inoltre introdotte le categorie di accordo e di 
ordine dei costituenti, che prima non erano state presentate e che quindi non sono comparabili con le altre ricerche. Non viene trattata separatamente la categoria della doppia negazione, già osservata in passato, in quanto questa tipologia di errore non raggiunge 1'1\%.

\section{CONCLUSIONI}

L'analisi dei problemi nella produzione scritta in italiano da parte di studenti slovenofoni all'esame di maturità professionale (livello A2/ B1) mette in evidenza come i problemi maggiori siano causati dalla categoria grammaticale dell'articolo, parte del discorso che lo sloveno non conosce. Seguono errori legati ai vari aspetti del verbo, e al terzo posto si colloca 1'uso delle preposizioni. Questi dati combaciano con quelli emersi dalle ricerche precedenti, le quali si sono occupate di L1 slavofoni (nella ricerca di Katerinov vengono inclusi nell'analisi tutti gli L1 slavi, in quella di Kruljac-Milani solo sloveni e croati). Pertanto, si può supporre che tutti i madrelingua slavi mostrino gli stessi problemi nell'apprendimento dell'italiano. Gli errori sono principalmente causati dal transfer negativo dalla L1 o altre L2/LS, ma anche da strategie di generalizzazione. Sono state notate delle interferenze con lo spagnolo, lingua genealogicamente più vicina all'italiano dell'inglese o del tedesco, il che rappresenta un'innovazione rispetto alle analisi svolte in passato.

Le osservazioni qui proposte andrebbero seguite aggiornando il corpus lavorativo annualmente, in modo da avere più dati e una maggiore rappresentatività, che possa sia confermare le tendenze individuate, sia trovarne di nuove. Questo è ciò che ci proponiamo di fare in lavori futuri.

Sarebbe auspicabile che i risultati qui proposti venissero presi in considerazione nella pianificazione didattica. Infatti, l'analisi contrastiva delle due grammatiche evidenzia i punti critici che, dopo un confronto con l'analisi degli errori, possono essere limitati solamente a quelli che in effetti si manifestano. Le discrepanze che non trovano riscontro in pratica, invece, vanno escluse. Si potrà così potenziare il lavoro su quelle veramente importanti a partire dalla progettazione del piano di- 
dattico, concepito come un unicum creato per ogni singolo destinatario in base al suo livello, alla L1, alle sue possibilità ed esigenze.

\section{BIBLIOGRAFIA}

Andorno, C. (2003). La grammatica Italiana. Milano: Mondadori.

Andorno, C. \& Nesci, M.T. (2004). Analizzare e correggere gli errori. Perugia: Guerra Edizioni.

Bažec, H. (2011). La nascita degli articoli nello sloveno. Retrieved from goo.gl/Bjkp2Y.

Bitenc Peharc, S. (2017). Umestitev nacionalnih izpitov iz italijanščine v skupni evropski jezikovni okvir. Retrieved from goo.gl/WKiEpZ.

Gaeta, L. (2010). Accordo. Enciclopedia Treccani. Retrieved from goo.gl/kM$v 64 s$.

Gass, S. \& Selinker, L. (2008). Second Language Acquisition: An Introductory Course. New York: Routledge.

Jessenr, U. (2008). Teaching third languages: Findings, trends and challenges. Language Teaching 41/1, 15-56.

Katerinov, K. (1980). L'analisi contrastiva e l'analisi degli errori di lingua applicata all'insegnamento dell'italiano a stranieri. Perugia: Edizioni guerra.

Mertelj, D. \& Premrl, M. (2013). Večstavčna skladnja pri pouku italijanščine Učenci med pozitivnim transferom in interferenco. Vestnik za tuje jezike 5, no. 1-2, 217-38.

Miklič, T. (2007). Metafore o načinih gledanja na zunajjezikovna dejanja v obravnavanju glagolskega vida. Slavistična revija, 35, no. 1-2, 85103.

Milani-Kruljac, N. (1990). La comunità italiana in Istria e a Fiume fra diglossia e bilinguismo. Trieste-Rovigno: Unione degli italiani dell'Istria e di Fiume, Università popolare di Trieste, Centro di ricerche storiche Rovigno.

Ožbot, M. (2009). Nekaj kontrastivnih beležk o italijanščini in slovenščini in nekaj opažanj o jezikovni produkciji pri govorcih slovenščine v Italiji. Jezik in slovstvo 54, no. 1, 25-47.

Salvi, G. \& Vanelli, L. (2004). Nuova grammatica italiana. Bologna: Il Mulino.

Selinker, L. (1972). Interlanguage. International Review of Applied Linguistics, 10, 209-41. 
Skela, J. \& Sešek, U. (2012). Od globali smernic do lokalnih kontekstov: učenje in poučevanje tujih jezikov v Sloveniji. Jezik in slovstvo 57, no. 3-4, 63-82.

Skubic, M. (1974). Il congiuntivo italiano delle opere letterarie contemporanee nelle traduzioni in sloveno. Linguistica, XIV, 77-95.

Smole, V. (2006). Lingvogeografska obdelava spola v ednini: samostalniki srednjega spola v ednini: samostalniki srednjega spola na $-\mathrm{o}$ v slovenskih narečjih. Slavistična revija, special edition, 125-36.

Toporišič, J. (2004). Slovenska slovnica. Maribor: Obzorja. Vallauri Lombardi, E. (2013). La linguistica in pratica Bologna: Il Mulino.

Riassunto: L'obiettivo del presente contributo è fare un'analisi degli errori su un corpus composto da 58 prove di produzione scritta in italiano all'esame di maturità professionale di studenti madrelingua sloveni a livello di competenza A2/B1. L'analisi qualitativa e quella quantitativa hanno l'intento di individuare gli errori più frequenti nel settore della morfosintassi, scoprirne le cause e compararli con i risultati emersi da ricerche simili svolte in precedenza. L'analisi qualitativa è stata effettuata in base a una griglia creata dopo uno studio contrastivo dei sistemi grammaticali dello sloveno e dell'italiano, nonché adattata a quanto realmente riscontrato nel corpus. Le principali cause degli errori risultano essere il transfer negativo dalla $\mathrm{L} 1 \mathrm{e}$ in una piccola percentuale da altre lingue straniere come l'inglese e lo spagnolo, ma anche la strategia della generalizzazione. Il più problematico quantitativamente risulta l'articolo, parte del discorso che lo sloveno non conosce, seguito da vari aspetti nella forma e nell'uso del verbo e dalla preposizione. Altre categorie che oltrepassano 1'1\% di tutti gli errori individuati e che quindi vengono incluse sono l'accordo tra i costituenti, l'ordine dei costituenti, errori legati a vari usi del pronome, dell'aggettivo, del nome e dell'avverbio. Questa frequenza di errori combacia con le altre ricerche simili svolte in passato, che però includevano studenti L1 appartenenti a diverse lingue slave. Questo fa presupporre che tutti i madrelingua slavi abbiano le stesse difficoltà, e quindi che le differenze nelle strutture grammaticali tra L1 e L2 siano la causa prima degli errori morfosintattici.

Parole chiave: analisi contrastiva, analisi degli errori, morfosintassi, italiano, sloveno 